\title{
Characteristic of no-synthase of peripheral blood lymphocytes of patients with rheumatic pathology
}

\author{
Ulyana Efremova, Nataliya Lychkovska, Roman Fafula, Zinoviy Vorobets
}

Danylo Halytsky Lviv National Medical University, Ukraine

\begin{abstract}
It is known that NO is a ubiquitous mediator which acts as a universal modulator of various functions in organism and is produced by three isoforms of NO synthase. Nowadays the role of NO in the development of autoimmune diseases is actively studied. However, it remains unclear the biochemical and biophysical mechanisms of disturbances of NOS activity in blood lymphocytes at autoimmune process. The aim of present work is to study the kinetic properties of NO-synthase of peripheral blood lymphocytes of patients with rheumatic pathology. The study was carried out on peripheral blood lymphocytes isolated from patients with rheumatoid arthritis and ankylosing spondylitis. NOS activity was determined on the saponin-permeabilized blood lymphocytes. The difference between the values of NADPH oxidation with L-Arg and with inhibitor L-NAME reflects the value of the NADPH oxidation, ie total NOS activity. The kinetic properties of NO-synthase in peripheral blood lymphocytes of patients with rheumatic pathology were studied. It was found that the development of rheumatic pathology is associated with an imbalance in the NO synthesis and changes of kinetic parameters of NOS. It was shown that reduction in eNOS activity is accompanied by a sharp increase in activity of its inducible form. It was established that inhibition of eNOS occurs by noncompetitive type. NO production in lymphocytes of patients with rheumatic diseases is mainly realized by iNOS, whereas under normal physiological conditions endothelial form of the enzyme is being involved.
\end{abstract}

Keywords: NO-synthase, nitric oxide, lymphocytes, rheumatoid arthritis, ankylosing spondylitis.

\section{Introduction}

At the end of the $20^{\text {th }}$ century it has been found that in any living organism a nitric oxide (NO) is produced in large concentrations. Further study of its biological role revealed that NO is a gaseous messenger which acts as a universal modulator of various functions in organism $[1,2]$. NO is a ubiquitous mediator that is produced by three isoforms of NO synthase (NOS): neuronal (nNOS I), endothelial (eNOS II) and inducible (iNOS III) [3]. Two isoforms of NO-synthase are classified as constitutive NO synthase - neuronal (nNOS I) and endothelial (eNOS II). Their designations indicate the cell types in which these isoforms have been identified. Functioning of constitutive forms is regulated by $\mathrm{Ca}^{2+}$ ions. eNOS is a $\mathrm{Ca}^{2+}$-dependent and after any stimulant which causes an increase in the intracellular $\mathrm{Ca}^{2+}$ concentration it synthesizes very small amounts of NO which has biomodulative effects. Also eNOS plays a key role in ensuring the constant "basal" NO level.

The third isoform of NO-synthase is inducible (iNOS II), as its activation induced by cytokines, endo- or exotoxins. Its synthesis occurs over 6-8 hours after their actions. iNOS is $\mathrm{Ca}^{2+}$-independent isoenzymes. In physiological conditions it is inactive. NO which is produced by the activation of inducible NOS is primarily designed for the protection of organism [4]. This isoform is associated with immunological and infectious stimuli and is expressed in macrophages, neutrophils and endothelial cells. The NO production by activated macrophag- 
es confirms a cytotoxic and cytostatic role of $\mathrm{NO}$ in immune system. It should thus be noted that NO can also be produced by non-enzyme interaction of arginine and hydrogen peroxide [5]. Also it has been found that eNOS produces low concentrations of NO, whereas iNOS synthesizes high concentrations of NO [4].

Although isoenzymes are products of different genes and they have different functions, the differentiation on inducible and constitutive NO synthesis is conditional, since they form a single product - a NO molecule, which diffuses easily through cell membranes and does not require receptors to perform its effects. The balance between physiological, regulatory and/or cytotoxic properties are largely due to the local concentration of $\mathrm{NO}$ and oxidative status of the tissue in which NO is synthesized and realizes its effects $[6,7]$.

Nowadays the role of $\mathrm{NO}$ in the development of autoimmune diseases is actively studied [8, 9]. It has been proved a key role of $\mathrm{NO}$ in the regulation of immune responses and its participation in almost every stage of inflammation. Inside the cells NO activates some enzymes and inhibits the others, thus participating in the regulation of cellular functions. High NOS activity leads to NO accumulation and initiation of pathological processes in the cell $[10,11]$. Herewith the inhibition of lymphocytes proliferation and increase of apoptosis of lymphocytes and macrophages occurs which leads to secondary immunodeficiency [12]. The NOS activity and NO level along with other parameters may indicate the state of the cell system and be auxiliary prognostic indicators for various diseases, including rheumatic.

Nowadays, a rheumatic diseases are considered as one of the most common pathologies of modern society. They are among the most difficult diseases by their severity and selection of adequate treatment. Most chronic inflammatory rheumatic diseases are mediated in a particular stage by immune disorders. The reason of immunocomplex destruction in patients with rheumatoid arthritis (RA) and ankylosing spondylitis (ASA) is the disturbances in the regulation of the immune response. This is due to imbalance in function of $\mathrm{T}$-and B-lymphocytes, which play an important role in compensatory-adaptive reactions of organism.

There is a large number of studies devoted to enzymatic spectrum of blood lymphocytes under various diseases, but the studies of NOS activity of blood lymphocytes in patients with RA and AS are limited.

As a result of our previous studies on the enzymatic activity of NOS in peripheral blood lymphocytes it was shown [13] that NOS activity in patients with RA and
ASA is significantly different from that in healthy persons. After the treatment the NOS activity in patients approaches to its control values. However, it remains unclear the biochemical and biophysical mechanisms of disturbances of NOS activity in blood lymphocytes at autoimmune process. The aim of this work was to study the kinetic parameters of NOS of peripheral blood lymphocytes of patients with RA and ASA.

\section{Materials and methods}

The study was carried out on peripheral blood lymphocytes isolated from patients with RA and ASA, treated in Lviv Regional Clinical Hospital (Ukraine). All patients were divided into two groups: patients with RA $(n=40)$ and patients with ASA $(n=30)$. Control group were practically (clinically) healthy persons representative by age and sex $(n=30)$. All patients and donors gave written informed consent to participate in research (Ethical Committee Approval Protocol No 8 from October 22, 2012).

\section{Isolation of lymphocytes}

Mononuclear peripheral blood lymphocytes were isolated from heparinized freshly obtained blood by ficoll-triombrast with gradient density $\rho=1.08 \mathrm{~g} / \mathrm{cm}^{3}$ [14]. Integrity and viability of blood lymphocytes evaluated using trypan blue staining in all experiments was at least $95 \%$ [15].

NOS activity was determined on the saponin-permeabilized blood lymphocytes. For permeabilization of lymphocytes membrane and disclosure of enzymatic activity lymphocytes were incubated for 10 min with moderate shaking in a solution containing saponin in a concentration of $0.2 \%[16,17]$. Protein content in lymphocytes mixture was determined by Lowry method [18].

\section{Determination of NOS}

Determination of NOS activity in permeabilized lymphocytes was carried out in the reaction mixture (at $37^{\circ} \mathrm{C}$ ) containing $80 \mathrm{mM}$ Tris- $\mathrm{HCl}$ buffer ( $\left.\mathrm{pH} 7.4\right), 5$ $\mathrm{mM} \mathrm{CaCl}{ }_{2}, 0.15 \mathrm{mM} \mathrm{L-Arg}, 0.12 \mathrm{mM}$ NADPH. The reaction was initiated by the introduction of an aliquot of blood lymphocytes $(70 \mu \mathrm{l})$ in the reaction mixture. Protein content in the sample did not exceed 50-75 $\mu \mathrm{g}$. The difference between the values of NADPH oxidation with L-Arg and with inhibitor L-NAME reflects the value of the NADPH oxidation, ie total NOS activity. The test samples were estimated spectrofotometrically against control samples at $340 \mathrm{~nm}$ and then incubat- 
ed for $20 \mathrm{~min}$ at $37^{\circ} \mathrm{C}$. The reaction was stopped by the introduction of $0.05 \mathrm{ml}$ of $1.5 \mathrm{M} \mathrm{HClO}_{4}$ and then decrease in extinction was registered [19]. NOS activity was expressed in nmol NADPH oxidase per 1 min per $1 \mathrm{mg}$ of lymphocytes protein [20].

The activity of $\mathrm{Ca}^{2+}$-independent iNOS was determined similarly, adding to the incubation medium $\mathrm{Ca}^{2+}$ chelators EGTA (4 mM) instead of $\mathrm{CaCl}_{2}$. The activity of $\mathrm{Ca}^{2+}$-dependent NOS isoforms was calculated as the difference between total NOS activity and $\mathrm{Ca}^{2+}$-independent NOS activity.

\section{Kinetic analysis}

Kinetic analysis of the enzyme reaction was performed in a standard incubation system (as described above) with modified physical and chemical characteristics or the respective components (the substrate concentration, incubation time, protein content and detergent concentration). The kinetic parameters characterizing the NO-synthase reaction - the initial (instantaneous) reaction rate $\left(V_{0}\right)$, maximum amount of the reaction product $\left(P_{\max }\right)$ and characteristic reaction time (time half saturation) $\tau$ were determined according to [21]. The apparent affinity constant for L-arginine $\left(K_{L \text {-Arg }}\right)$ and maximum reaction rate $\left(V_{\max }\right)$ were determined by Lineweaver-Burk plot [22]. Kinetic and statistical calculations were carried out using the software MS Office computer programs. The results were treated by methods of variation statistics using Student $t$-test. The equation of the straight line that approximates the experimental data the best was calculated by method of least squares. The absolute value of the correlation coefficient $r$ was from 0.85 to 0.95 . The significance of the calculated parameters of line was tested by the Fisher's F-test. The accurate approximation was when $P \leqslant 0.05$.

\section{Results}

Kinetic analysis of NOS activity on concentration of L-arginine

Isoenzymes of NOS are dioxygenases which use molecular oxygen and NADPH for the transformation of L-arginine to L-citrulline and NO. Arginine is the basic amino acid substrate for NO production by all NOS isoforms.

For this reason, changes in the L-arginine concentration in the incubation medium affect the rate of NOsynthase reaction. The dependence of the NO-synthase activity on substrate concentration in the incubation medium is determined by the apparent affinity con- stant to the substrate $K_{\text {Larg }}$. For its determination the $L$-arginine was added to the incubation medium in concentrations ranging from 0.1 to $30 \mathrm{mM}$ (at constant concentration of $\mathrm{CaCl}_{2}-10 \mathrm{mM}$ and NADPH - 0.12 $\mathrm{mM})$. It was observed a monotonic increase in the enzymatic activity of two isoforms of NOS reaching a plateau (Figure 1). As can be seen from figure 1 the eNOS activity in patients with RA is reduced in comparison with the value in healthy donors in the whole range of $L$-arginine concentrations. Reduction of eNOS activity is accompanied by a sharp increase in activity of inducible form. The optimum substrate concentration for both enzymes is within 20-30 $\mu \mathrm{M}$. The dependence NOS activity on L-arginine concentration for patients with ASA has an identical character.

It was determined the main kinetic parameters of L-arginine hydrolysis to elucidate the possible mechanism of change in NOS activity in patients with RA and ASA (Figure 2).

Linearization of concentration curves in Lineweaver-Burk plot for iNOS has an identical character. The main kinetic parameters NOS of blood lymphocyte of donors and patients with RA and ASA were calculated by linearization of the data in Lineweaver-Burk plot (Table 1).

Data in table 1 show that the maximum rate of hydrolysis of L-arginine for eNOS of blood lympho-

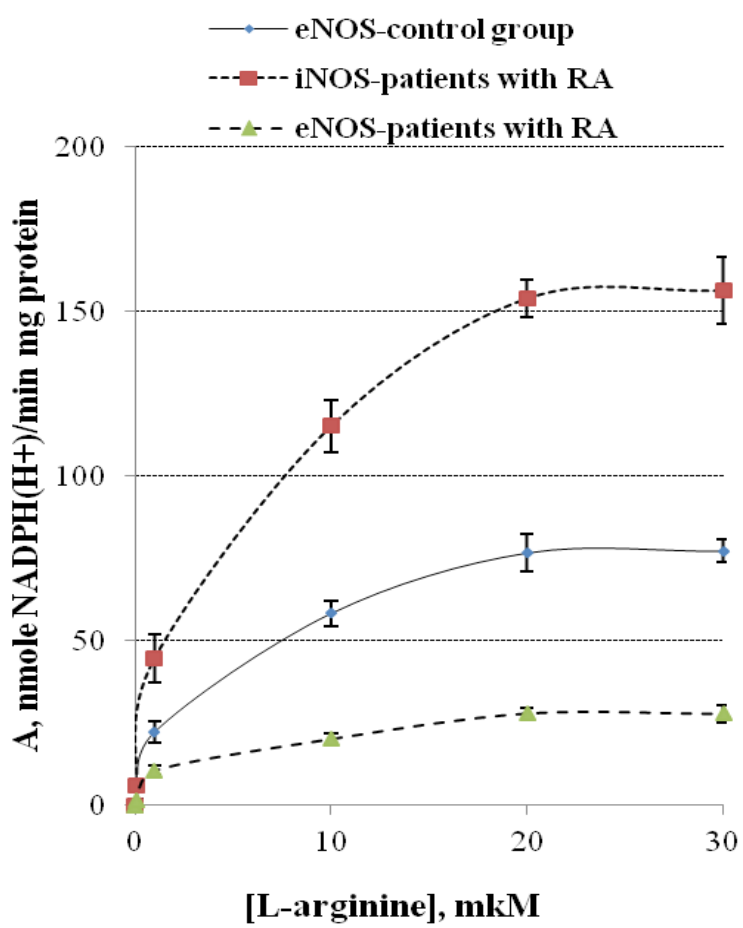

Figure 1. Concentration dependence of L-arginine effect on NOS activity of saponin-permeabilized peripheral blood lymphocytes of patients with rheumatoid arthritis, $M \pm m, n=6-8$ 


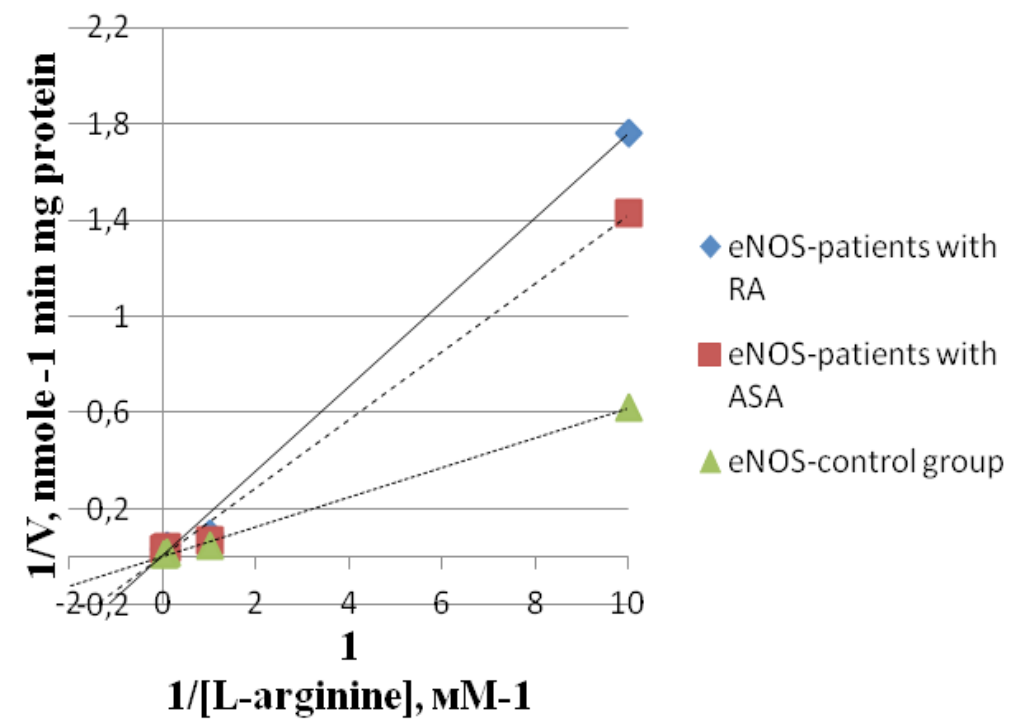

Figure 2. Linearization of concentration curves in Lineweaver-Burk plot, here V is eNOS activity of saponin--permeabilized peripheral blood lymphocytes of patients with rheumatoid arthritis and ankylosing spondylitis, $n=4-6 ; r>0.85 ; F<0.005$

Table 1. Kinetic parameters of NOS of blood lymphocytes of patients with RA and ASA, defined by L-arginine, M $\pm m, n=6-8$

\begin{tabular}{|c|c|c|c|}
\hline Kinetic parameters & Patients with RA & Patients with ASA & Control group \\
\hline & & \multicolumn{2}{|c|}{ eNOS } \\
\hline$V_{\max }, \mathrm{nmol} \mathrm{NADPH}\left(\mathrm{H}^{+}\right) / \mathrm{min} \cdot \mathrm{mg}$ protein & $92.2 \pm 12.8^{\star * \star}$ & $108.8 \pm 8.2^{* \star *}$ & $235.6 \pm 44.0$ \\
\hline \multirow[t]{2}{*}{$K_{\text {L-Arg' }} \mu \mathrm{M}$} & $16.3 \pm 0.4$ & $15.8 \pm 1.5$ & $14.6 \pm 2.7$ \\
\hline & & \multicolumn{2}{|c|}{ iNOS } \\
\hline$V_{\max }, \mathrm{nmol} \mathrm{NADPH}\left(\mathrm{H}^{+}\right) / \mathrm{min} \cdot \mathrm{mg}$ protein & $164.3 \pm 10.2$ & $142.4 \pm 11.0$ & - \\
\hline$K_{\mathrm{L}-\mathrm{Arg}}, \mu \mathrm{M}$ & $2.8 \pm 0.5$ & $2.6 \pm 0.4$ & - \\
\hline
\end{tabular}

cytes of healthy persons is 2.5 and 2.2 times greater than this value for eNOS of patients with RA and ASA accordingly. However, affinity constants for $L$-arginine in all studied groups were not significantly different. Hence we can conclude that affinity of eNOS for L-arginine does not change in patients with RA and ASA. Thus, in patients with rheumatic pathology the inhibition of eNOS in immunocompetent cells occurs by noncompetitive type, by reducing the speed of the enzyme (value of $V_{\max }$ decreases).

The maximum rate of L-arginine hydrolysis for iNOS (which is not identified in normal and is significantly activated in rheumatic diseases) differs from this value for eNOS of blood lymphocytes in healthy persons (in 1.4 times greater than in patients with RA and 1.6 times greater than in patients with ASA) Affinity constant for L-arginine for iNOS of blood lymphocytes in patients with rheumatic disorders is lower than for eNOS in healthy persons (in 5.2 times in patients with RA and in 5.6 times in patients with ASA).
Kinetic analysis of NO-synthase reaction on time For studying the peculiarities and the mechanism of NOS functioning the initial (instantaneous) reaction rate $\left(V_{0}\right)$, maximum amount of the reaction product $\left(P_{\max }\right)$ and characteristic reaction time (time half saturation) $(\tau)$ were determined [21]. For determination of these kinetic parameters of NOS the dynamics of $\mathrm{NADPH}\left(\mathrm{H}^{+}\right)$accumulation, indicating NO synthesis, was examined. Suspension of lymphocytes was incubated in the standard incubation medium for various periods of time $(0-30$ min.). These experiments show that kinetics of NO-synthase reaction by saponin-permeabilized lymphocytes is reflected by curves that tend to saturation (Figure 3).

Analysis of the results shows that kinetics of NO production by eNOS is consistent with the first-order reaction in the range $0-20 \mathrm{~min}$. In this time interval the dependence of NO production on the incubation period is almost linear. Therefore, in further experiments the incubation time of lymphocytes and, therefore, NO-synthase reaction is $20 \mathrm{~min}$. 


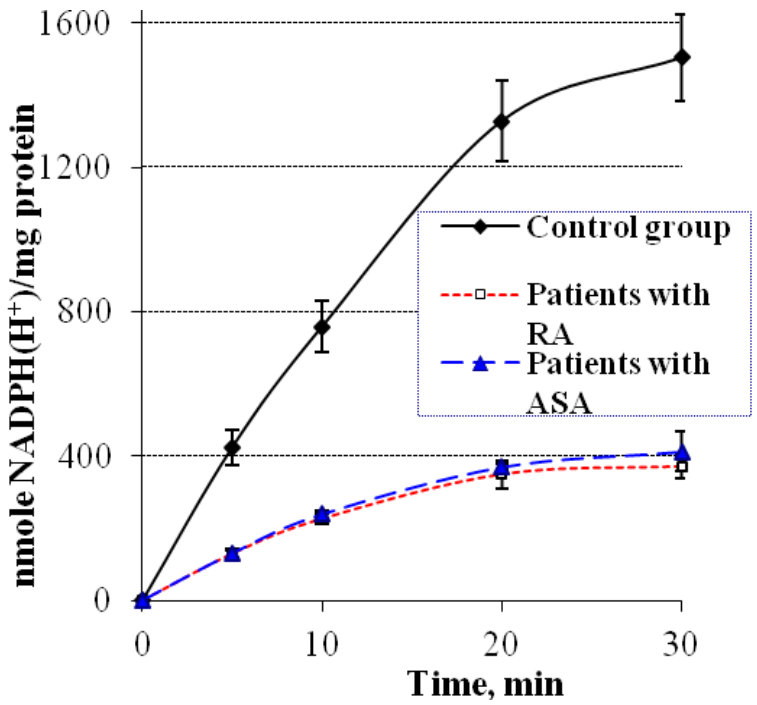

b

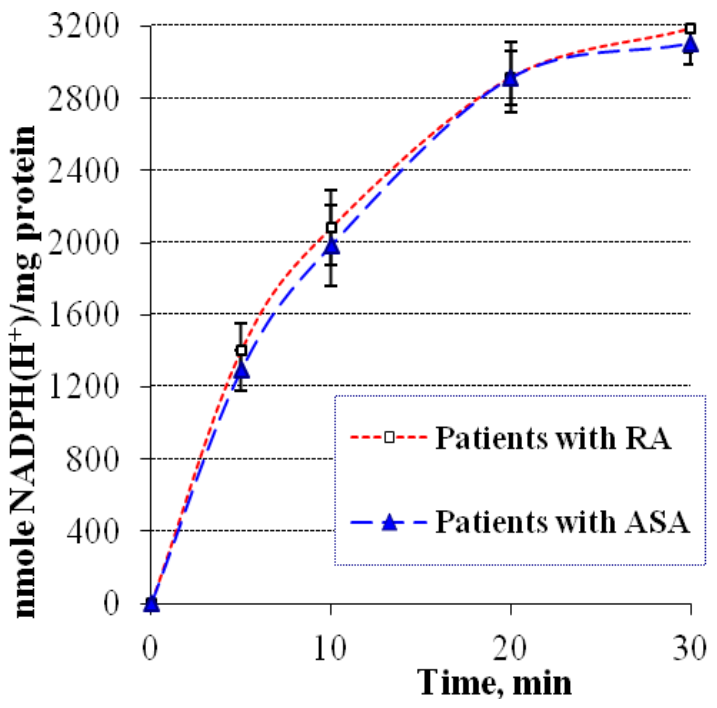

Figure 3. Dynamics of NADPH(H+) production in NOS reaction of blood lymphocytes by eNOS (a) and by iNOS (b) in patients with RA and ASA, M $\pm m$, $n=6-8$

As can be seen from Figure $3 a$ in the whole range of time, the value of NO produced by eNOS of blood lymphocytes of patients with rheumatic diseases is much lower compared to value in healthy donors. However, the value of NO produced by iNOS (Figure 3 b) of blood lymphocytes of patients with rheumatic diseases is significantly higher than these values in donors.

By linearization of the data in the coordinates $P / t$ on $P$ the main kinetic characteristics of eNOS and iNOS of blood lymphocytes in patients with RA were calculated (Table 2, Figure 4). In patients with ASA the linearization curves of NADPH accumulation by endothelial and inducible form of NOS have a similar appearance.

As can be seen from Table 2 the values of the kinetic parameters of NO production by eNOS of blood lymphocytes of patients with rheumatic diseases and healthy persons differ significantly. The maximum instantaneous rate of eNOS is greater in 3 times in healthy donors compared with values in patients with RA and ASA. Maximum amount of reaction product by eNOS in control group exceeds this value in patients with RA and ASA in 5.1 and 4.2 times accordingly.

The results of kinetic analysis indicate that NO production by iNOS is much more intense than by eNOS, and, in turn, NO production by eNOS in the control group is quicker and more active than in patients with rheumatic pathologies. The maximum instantaneous rate of iNOS reaction exceeds this value of eNOS reaction in patients with RA and ASA in 12 and 10.8 times accordingly. Maximum amount of reaction product by iNOS exceeds this value for eNOS in patients with RA and ASA in 6.5 and 5.7 times respectively.

\section{Kinetic analysis of NOS activity on concentration} of protein

Taking into account that enzyme activity depends on the protein content in incubation medium, the NOS

Table 2. Kinetic parameters of L-arginine hydrolysis by NOS of blood lymphocytes of patients with RA and ASA, M $\pm m, n=6-8$

\begin{tabular}{lccc}
\hline \multicolumn{1}{c}{ Kinetic parameters } & Patients with RA & Patients with ASA & Control group \\
\hline & & eNOS & \\
\hline$V_{0}, \mathrm{nmol} \mathrm{NADPH}\left(\mathrm{H}^{+}\right) / \mathrm{min} \cdot \mathrm{mg}$ protein & $33.9 \pm 2.4^{* * *}$ & $32.8 \pm 2.7^{* * *}$ & $98.0 \pm 7.2$ \\
\hline$P_{\max ,} \mathrm{nmol} \mathrm{NADPH}\left(\mathrm{H}^{+}\right) / \mathrm{mg}$ protein & $690.1 \pm 83.0^{* * *}$ & $827.6 \pm 91.0^{* * *}$ & $3512 \pm 306$ \\
\hline$\tau, \min$ & $21.4 \pm 5.9^{* *}$ & $26.0 \pm 6.8^{* * *}$ & $36.6 \pm 4.6$ \\
\hline & & iNOS & - \\
\hline$V_{0,} \mathrm{nmol} \mathrm{NADPH}\left(\mathrm{H}^{+}\right) / \mathrm{min} \cdot \mathrm{mg}$ protein & $405.2 \pm 50.3$ & $356.0 \pm 37.4$ & - \\
\hline$P_{\max } \mathrm{nmol} \mathrm{NADPH}\left(\mathrm{H}^{+}\right) / \mathrm{mg}$ protein & $4524.2 \pm 282.6$ & $4725.9 \pm 302.8$ & - \\
\hline$\tau, \min$ & $11.6 \pm 1.9$ & $13.7 \pm 2.1$ & \\
\hline$* * P<0.01$ & & & \\
$* * * P<0.001$ compared to healthy donors & & &
\end{tabular}


reaction was initiated by inclusion of protein with concentrations ranging from 25 to $150 \mu \mathrm{g} / \mathrm{ml}$ in lymphocyte mixture (Figure 5). It was investigated that a gradual increase in lymphocyte protein concentration in the incubation medium leads to an increase in $V_{0}$ of NOS reaction.
The dependence of the $\operatorname{NADPH}\left(\mathrm{H}^{+}\right)$production on the protein content in incubation medium has the same character both for eNOS and iNOS. However, the data indicate that maximum instantaneous rate of $\mathrm{NADPH}\left(\mathrm{H}^{+}\right)$ production by eNOS in patients with RA and ASA is significantly different from that of healthy donors (Table 3 ).

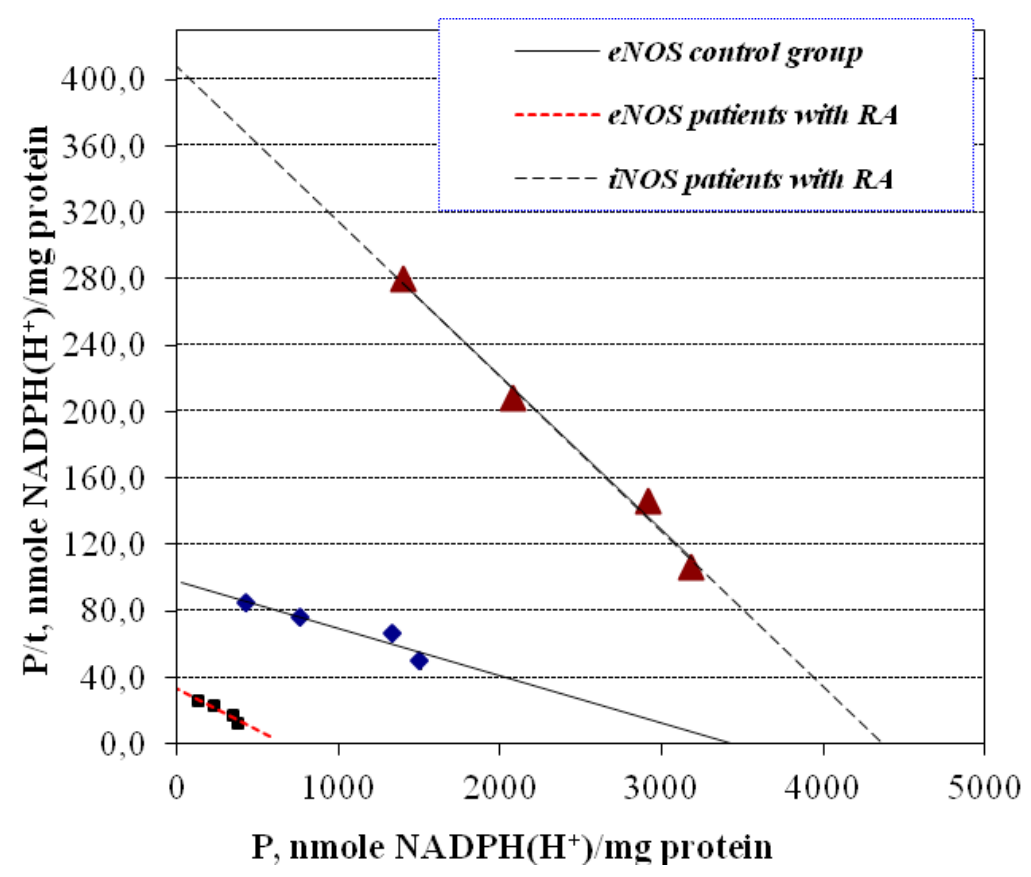

Figure 4. Linearization of curves of $\mathrm{NADPH}(\mathrm{H}+)$ accumulation by eNOS and by iNOS of saponinpermeabilized peripheral blood lymphocytes of patients with rheumatoid arthritis in coordinates $[P / t ; P], n=6-8 ; r>0.9 ; F<0.02$

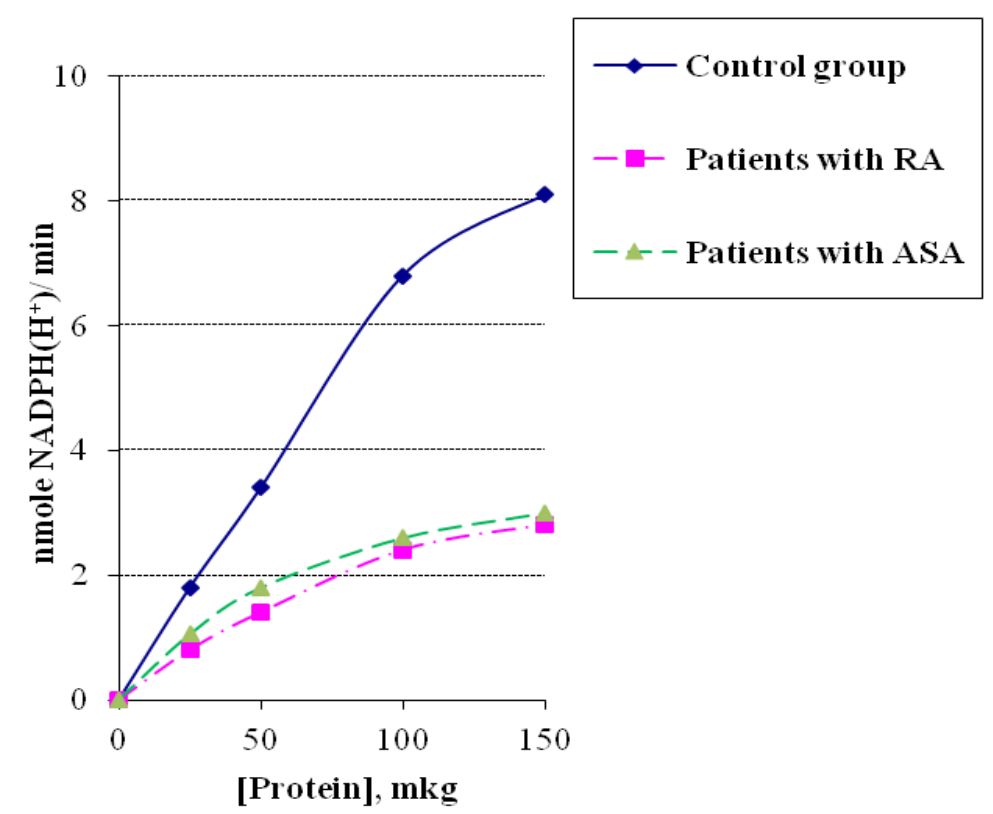

Figure 5. Dependence of the initial rate of eNOS reaction of saponin-permeabilized blood lymphocytes of patients with RA and ASA on protein content, $M \pm m, n=4-6$ 
Table 3. The kinetic parameters of $\mathrm{NADPH}\left(\mathrm{H}^{+}\right)$production by eNOS of saponin-permeabilized blood lymphocytes of patients with $R A$ and $A S A, M \pm m, n=4-6$

\begin{tabular}{lccc}
\hline \multirow{2}{*}{ Kinetic parameters } & \multirow{2}{*}{ Control group } & \multicolumn{2}{c}{ Patients with } \\
\cline { 3 - 4 } & & RA & ASA \\
\hline$V_{0,} \mathrm{nmol} \mathrm{NADPH}\left(\mathrm{H}^{+}\right) /$min $\cdot \mathrm{mg}$ protein & $53.6 \pm 1.0$ & $18.6 \pm 0.5^{* * *}$ & $17.6 \pm 1.4^{* * *}$ \\
\hline${ }_{* * *} P<0.001$ compared to healthy donors & & &
\end{tabular}

Analysis of the dependence of NOS activity on the detergent (saponin) concentration

Studying the NOS activity the different methodological approaches (studies on isolated subcellular structures, on whole cells or on cell homogenates) are used. Study of the NOS properties on isolated membrane fractions or lymphocyte mitochondria is difficult due to their gradual inactivation during preparative obtain of fractions of subcellular structures.

The aim was to pick up the optimal conditions for NOS determination using saponin as a detergent. This substance is capable of binding to membrane proteins by hydrophobic bonds, while interacting by polar groups with water. This allows molecules of detergent to loosen the membrane without disrupting its structure and function [31]. Saponin was used in the concentration range from 0.02 to $0.3 \%$.

As can be seen from Figure 6 in a wide range of saponin concentrations (0.02-0.3\%) curves of enzyme activity on the detergent concentration are dome shaped. The maximum of NOS activities is observed for saponin concentrations of $0.1-0.2 \%$. This range of saponin concentrations can be considered as the most appropriate for practical use in experiments to study the kinetic and catalytic properties of NOS. Lower saponin concentrations $(0.02-0.04 \%)$ do not disclosure of NOS activity fully. Dependences NOS activity on saponin concentration for lymphocytes of patients with ASA have a similar appearance.

\section{Discussion}

The availability of $L$-arginine is a potential mechanism for controlling the NO production, since most cell types can not synthesize arginine and require arginine intake from outside [23, 24, 25].

Physiological needs of most tissues and organs of mammals in arginine are satisfied with its endogenous synthesis and/or intake of food. But this amino acid is essential for young individuals and adults under stress or illness. Arginine is an essential precursor for the synthesis of proteins and many biologically important molecules, such as ornithine, proline, polyamines, creatine and agmatine. However, the main role of arginine in organism is to be a substrate for NO synthesis [26].

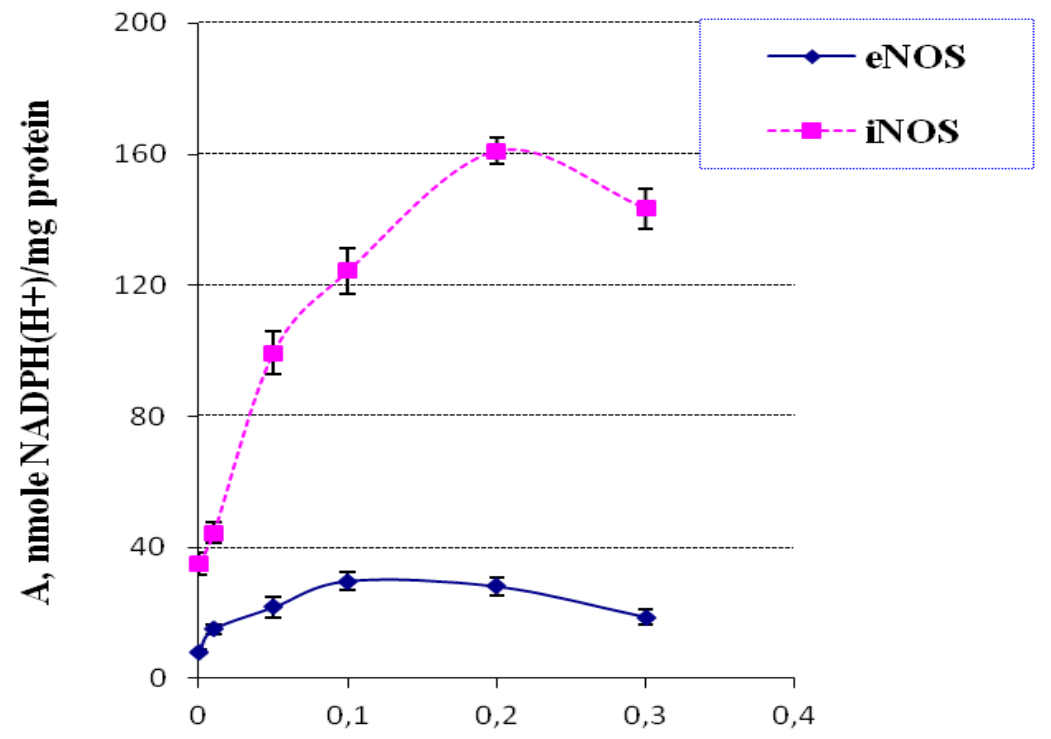

[Saponin], \%

Figure 6. The dependence of eNOS and iNOS activity of saponin-permeabilized blood lymphocytes of patients with RA on saponin concentration, $M \pm m, n=4$ 
However, local bioavailability of L-arginine for NOS may be reduced due to the increased activity of L-arginase, which competes with NOS for the same substrate. Arginases metabolize arginine to ornithine and urea $[23,27,28]$.

If iNOS is induced by classically inflammatory cytokines (IL-1, TNF- $\alpha, \gamma$ and IFN- $\gamma$ and IL-2), the humoral proinflammatory cytokine (IL-4, IL-10, IL-13 and TGR- $\beta$ ) induce the expression of arginase. Endotoxin induces both iNOS and arginase I [27]. iNOS provides a regulating effect on arginase activity through production of hydroxy-L-arginine which is an intermediate product in the NO production. Arginase, in turn, can regulate NO synthesis through depletion of arginine availability [28].

Taking into account that cellular NO production in immune cells is completely dependent on the presence of L-arginine [29], a sharp increase in iNOS activity in blood lymphocytes of patients with RA and ASA is probably associated with an increase in substrate concentration.

Based on our data, we assume that in white blood cells of patients with RA and ASA the NO production by iNOS is much more intense than by eNOS. NO production by eNOS in patients with rheumatic pathology is slower and less active than in healthy donors. Thus, we can conclude that in lymphocytes of patients with RA and ASA the NO production is realized by inducible isoform of NOS mainly, and under normal physiological conditions involving endothelial enzyme form.

Using whole cells the different NOS isoforms are in latent state and inaccessible to substrates. Therefore, testing their activities possible after prior disturbance of integrality of lymphocytes plasma membranes. This can be achieved by introducing a substance leading to perforation of plasma membranes (detergent) in the incubation medium. Using a suspension cells pretreated with detergent is adequate for correct testing of NOS of subcellular structures. Under these conditions the natural interrelation of intracellular stores is obey [30].

Similar studies of dependence of enzyme activity on the saponin concentration were carried out by other researchers. It was shown that similar saponin concentrations are used to uncover latent activity of bot h membrane and cytosolic enzymes [31]. Using such methodological approach for testing the enzymes is also confirmed by ultrastructural study [32].

Under conditions of rheumatic pathologies the effects of NO are different. On the one hand, a disturbance of constitutive NO production is associated with a decrease in the eNOS activity. On the other hand, these conditions can lead to iNOS hyperactivation and excess production of cytotoxic amounts of NO.

\section{Conclusions}

It was shown that reduction in eNOS activity is accompanied by a sharp increase in activity of its inducible form. It was established that inhibition of eNOS occurs by noncompetitive type. NO production in lymphocytes of patients with rheumatic diseases is mainly realized by iNOS, whereas under normal physiological conditions endothelial form of the enzyme is being involved.

\section{Acknowledgements}

\section{Conflict of interest statement}

The authors declare that there is no conflict of interest in the authorship or publication of contribution.

\section{Funding sources}

There are no sources of funding to declare.

\section{References}

1. Gurin AV. The functional role of nitric oxide in the central nervous system. Successes of physiological Sciences. 1997;28(1):53-60.

2. Markov HM. Nitrogen oxides and carbon oxide as a new class of signaling molecules. Successes of physiological Sciences. 1996;27(4):30-43.

3. Griffith O, Stuehr D. NO-synthases: properties and catalytic mechanism. Ann Rev Physiol. 1995;57:707-736.

4. Kostogryz VB, Turovskaya TV. Peculiarietis of nitric oxide metabolism in patients with chronic heart failure of ischemic etiology with an implanted pacemaker: possible drug correction by beta-adrenoceptor blockers. Ukrainian Medical Journal. 2010;5(79):81-84.

5. Tse WY, Williams J, Pall A, Wilkes M, Savage CO, Adu D. Antineutrophil cytoplasm antibody-induced neutrophil nitric oxide production is nitric oxide synthase independent. Kidney Int. 2001 Feb; 59(2):593-600.

6. Korhonen R, Lahti A, Kankaanranta H, Moilanen E. Nitric oxide production and signaling in inflammation. Curr Drug Targets Inflamm Allergy. 2005 Aug;4(4):471-479.

7. Mannick JB. Immunoregulatory and antimicrobial effects of nitrogen oxides. Proc Am Thorac Soc. 2006 Apr; 3(2):161-165.

8. Loveless MO, Phillips CR, Giraud GD, Holden WE. Decreased exhaled nitric oxide in subjects with HIV Infection. Thorax. 1997 Feb;52(2):185-186.

9. Rolla G, Brussino L, Bertero MT, Colagrande P, Converso M, Bucca C, Polizzi S, Caligaris-Cappio F. Increased nitric oxide in exhaled air of patient with systemic lupus erythematoses. J Rheumatol. 1997 Jun;24(6):1066-1071.

10. Bredt DS. Molecular characterization of nitric oxide synthase. In: Nitric oxide in the nevous system. Ed. Vincent SR. New York: Academic Press; 1995; 1-21. 
11. Garthwaite J. Neural nitric oxide signalling. Trends Neurosci. 1995;18(2):51-56.

12. Sarih M, Souvannavong V, Adam A. Nitric oxide synthase induced macrophage death by apoptosis. Biochim Biophys Res Comm. 1993;193(3):503-507.

13. lefremova UP, Lychkovska NE, Fafula RV, Vorobets Z. Disturbances of metabolism of nitric oxide under rheumatoid arthritis and its correction. Journal of Lugansk university. 2012;17:52-58.

14. Boyum A. Isolation of mononuclear cells and granulocytes from human blood. Scand J Clin Lab Invest. 1968;21(97):77-79.

15. Mishell BB, Shiigi SM, Freeman WH. Selected Methods in Cellular Immunology. WH. Freeman and Company, San Francisko; 1980; 486.

16. Kocheshkova NS, Vorobets ZD. Eosin-sensitive ATPase activity in spermatozoa of men as a biochemical test for oligozoospermia. Ukrainian Biochemical Journal. 2007;79(2):45-55.

17. Kimakovich OV, Pidkovka NO, Vorobets ZD. Action of kvamatel and pirenzepine on transport ATPase activity of peripheral blood lymphocytes. Practical Medicine. 2004;10(2):86-89.

18. Lowry OH, Rosebrough NJ, Farr AL. Protein measurement with the Folin phenolreagent. J Biol Chem. 1951;193:265275.

19. Sybirna NO, Maevska OM, Barska ML. Investigation of some biochemical parameters under oxidative stress condition. Lviv: Vydav. Centr Ivana Franka; 2006; 60.

20. Sagach B, Prisyajna O, Tkachenko M. Effect of L-arginine on endothelial functional activity in experimental diabetes. Physiological Journal. 2005;51(2):3-7.

21. Kosterin SA, Burchinskaya NF. Method for determination of kinetic parameters of $\mathrm{Ca} 2+$-transporting systems of subcellular structures of smooth muscle. Ukrainian Biochemical Journal. 1987;59(2):66-69.

22. Keleti T. Fundamentals of enzyme kinetics. Moscow: Mir; 1990; 350 .

23. Dmitrenko NP, Kyshko TO, Shandrenko HS. Arginine: biological action, effect on nitric oxide synthesis. Ukrainian chemotherapeutic Journal. 2008;1-2(22):137-140.

24. Morris CR, Morris SM Jr, Hagar W, Van Warmerdam J, Claster S, Kepka-Lenhart D et al. Arginine therapy: a new treatment for pulmonary hypertension in sickle cell disease? Am J Respir Crit Care Med. 2003 Jul 1;168:63-69.
25. Popovic PJ, Zeh HJ, Ochoa JB. Arginine and Immunity. The J of Nutr. 2007;137:1687-1692.

26. Boger RH. The pharmacodynamics of L-arginine. J Nutr. 2007 Jun; 137:1650-1655.

27. Chatterjee S, Premachandran S, Bagewadikar RS, Bhattacharya S, Chattopadhyay S, Poduval TB. Arginine metabolic pathways determine its therapeutic benefit in experimental heatstroke: role of Th1/Th2 cytokine balance. Nitric Oxide. 2006;15:408-416.

28. Morris SMJr. Enzymes of arginine metabolism. J Nutr 2004;134(10):2743-47.

29. Mori M, Gotoh T. Regulation of nitric oxide production by arginine metabolic enzymes. Biochem Bioph Res Commun. 2000 Sep 7;275:715-719.

30. Shlykov SG, Babich LG, Kosterin SA. The suspension of smooth muscle cells treated with digitonin solution, as a model for studying the calcium pump of the endoplasmic reticulum of myometrium. Biochemistry. 1997;62(12):1666-1671.

31. Pidkovka NO, Vorobets ZD, Zimenkovsky AB. Study of some properties of ATPase in human blood lymphocytes. Experimental and clinical physiology and biochemistry. 2002;7(1):38-41.

32. Fafula RV, Efremova UP, Lychkovska NE, Melnyk OV, Vorobets ZD, Kulachkovskuy OR. The methodological approach to the study of enzymatic spectrum lymphocytes at pathological conditions using detergent saponin (ultrastructural study). Bulletin of the problems of biology and medicine 2012;1(96):163-166.

Acceptance for editing: 2015-02-4 Acceptance for publication: 2015-03-30

Correspondence address: Ulyana Efremova Department of Medical Biology Danylo Halytsky Lviv National Medical University 69 Pekarska Str., 79010 Lviv, Ukraine phone: + 380930390437 fax: +380322757632 e-mail: efremova_ulyana@ukr.net 